\title{
Valorisation de la diversité génétique du sorgho par des approches de sélection participative au Nicaragua
}

\author{
Gilles Trouche $e^{1,2}$ \\ Silvio Aguirre Acuña ${ }^{3}$ \\ Henri Hocdé ${ }^{4}$ \\ Rafael Obando Solís ${ }^{5}$ \\ Zildghean Chow Wong ${ }^{6}$ \\ ${ }^{1}$ Centre de coopération internationale \\ en recherche agronomique \\ pour le développement (Cirad), \\ Département Systèmes biologiques, \\ Unité de recherche (UR) «Agrobiodiversité \\ des plantes de savane ", \\ Avenue Agropolis, \\ 34398 Montpellier cedex 5 \\ < gilles.trouche@cirad.fr> \\ ${ }^{2}$ Centro international de agricultura tropical \\ (CIAT), \\ A.A. 6713, \\ km 14 Autopista Cali-Palmira, \\ Cali Colombie \\ <g.trouche@cgiar.org> \\ ${ }^{3}$ Centro para la investigación, la promoción y \\ el desarrollo rural y social (CIPRES), \\ Pueblo Nuevo \\ Nicaragua \\ <silvioacua@yahoo.com> \\ ${ }^{4}$ Centre de coopération internationale \\ en recherche agronomique \\ pour le développement (Cirad), \\ Département Environnements et sociétés, \\ Unité de recherche (UR) "Actions \\ collectives, politiques et marchés " \\ 73, rue Jean-François Breton, \\ 34398 Montpellier cedex 5 \\ <henri.hocde@cirad.fr> \\ ${ }^{5}$ Instituto nicaragüense de tecnología \\ agropecuaria (INTA), \\ CNIA Km. 14.5, \\ Carretera Norte, \\ Managua \\ Nicaragua \\ ${ }^{6}$ Projet Centro international de agricultura \\ tropical (CIAT)-Centre de coopération \\ internationale en recherche agronomique \\ pour le développement (Cirad), \\ Residencial Los Robles, \\ Casa 303, \\ LM 172, \\ Managua \\ Nicaragua \\ <ciatarroz@cablenet.com.ni>
}

Tirés à part : G. Trouche

\begin{abstract}
Résumé
Au Nicaragua, le Centre de coopération internationale en recherche agronomique pour le développement (Cirad) et le Centro international de agricultura tropical (CIAT) ont conduit entre 2002 et 2006 un projet de recherche sur la sélection participative du sorgho destiné aux petits producteurs des zones sèches. Ce projet a été mis en œuvre dans deux régions, en collaboration avec la recherche agricole nationale, des organisations non gouvernementales (ONG) et des organisations locales de producteurs. En ayant largement recours à du germoplasme africain, la stratégie appliquée de sélection variétale participative et décentralisée a permis d'identifier différentes lignées bien adaptées aux systèmes de culture locaux, apportant des gains significatifs en rendement et précocité par rapport aux cultivars locaux et avec une qualité de grain appropriée pour l'élaboration des tortillas et autres spécialités alimentaires locales. Par rapport à une approche conventionnelle centralisée, cette stratégie permet de diffuser plus rapidement une nouvelle variété avec de plus grandes chances d'adoption massive par les agriculteurs. En outre, elle contribue à accroître la diversité génétique et le nombre des variétés disponibles pour ces agrosystèmes vulnérables, à faibles intrants. Les schémas en cours de création variétale participative in situ annoncent à moyen terme du matériel génétique prometteur, plus performant et mieux adapté aux divers objectifs de production.
\end{abstract}

Mots clés : amélioration des plantes ; approches participatives ; Nicaragua ; organisation paysanne ; ressource génétique ; sorgho.

Thèmes : productions végétales ; ressources naturelles et environnement ; systèmes agraires.

\section{Abstract \\ Valorisation of genetic diversity of sorghum through participatory breeding in Nicaragua}

In Nicaragua, the Centre de coopération internationale en recherche agronomique pour le développement (Cirad) together with the Centro international de agricultura tropical (CIAT) conducted a research project from 2002 to 2006 on participatory breeding of sorghums addressed to small farmers located in dry areas. This project was implemented in two regions in collaboration with national agricultural research, several NGOs and local farmers' organizations. With a wide utilization of African germplasm, the applied strategy of decentralized participatory variety selection among inbred genetic materials made it possible to identify various lines well-adapted to local cropping systems, bringing significant gains in yield and earliness compared with local cultivars and with adequate grain quality for making tortillas and other local dishes. In comparison with a conventional centralized program, this strategy makes it possible to diffuse a new variety more rapidly with a higher probability of large-scale adoption by farmers. Furthermore, it contributes to enlarging genetic diversity and the number of varieties available for these vulnerable low input agro-systems. The current in situ participatory breeding schemes display new and promising genetic materials for medium term with better agronomic performance and responses to diverse production goals.

Key words: farmers organizations; genetic resources; Nicaragua; participatory approaches; plant breeding; sorghum grain.

Subjects: farming systems; natural resources and environment; vegetal productions. 
A u Nicaragua, le sorgho n'occupe que le troisième rang des cultures céréalières avec environ 75000 hectares emblavés annuellement (Instituto nacional de estadisticas y censos de Nicaragua, 2002). Cependant, dans les régions sèches, où la production de maïs est insuffisante et aléatoire, les sorghos à grain blanc représentent une culture de première importance dans les agrosystèmes à faibles intrants et jouent un rôle essentiel pour la sécurité alimentaire des petits producteurs et de leurs familles. On y distingue deux types de sorgho intégrés dans des systèmes de culture distincts :

- millón: sorgho photopériodique de cycle long semé en association avec le maïs ou le haricot dans des systèmes de culture manuels, principalement semé en zones de collines;

- tortillero : sorgho non photopériodique de cycle court, généralement semé en culture pure dans des systèmes de culture semi-intensifiés en zones planes (vallées et plaines).

Pour les petits producteurs, le sorgho à grain blanc est une plante à usages multiples. Les grains sont utilisés pour l'élaboration des tortillas (galettes) et d'autres plats traditionnels et pour l'alimentation des animaux de la ferme (volailles et porcs). Les pailles sont largement utilisées ou vendues pour servir de fourrage en saison sèche. L'irrégularité et l'insuffisance des pluies, la faible fertilité des sols et l'utilisation limitée des engrais, les dégâts de certains insectes ravageurs et des maladies sont les principaux facteurs limitant la productivité de ces sorghos dans la frange Trópico Seco du Nicaragua. De plus, les rares variétés améliorées adaptées à ces conditions, ne présentent pas les qualités de grain et de paille requises pour répondre pleinement aux différents usages.

Dans ce contexte, le Centre de coopération internationale en recherche agronomique pour le développement (Cirad) et le Centro international de agricultura tropical (CIAT) ont mis en ouvre depuis 2002 le projet "Sélection participative des sorghos ", en collaboration avec l' Instituto nicaragüense de tecnología agropecuaria (INTA, Institut nicaraguayen de technologies agricoles), des organisations non gouvernementales (ONG) nationales et des organisations paysannes locales. Sur la base d'expériences précédentes de recherche participative conduites dans la région, le projet s'est donné comme principe de travailler dès le début avec des groupes d'agriculteurs déjà organisés au niveau de leur communauté et, pour la plupart, déjà formés à l'expérimentation agronomique. Ce projet est essentiellement conduit dans deux régions sèches de la Cordillère centrale, au nord (départements de Madriz et Esteli) et au centre (département de Matagalpa), en intervenant dans plusieurs villages-sites par région.

Dans sa phase initiale (2002-2003), l'équipe du projet a conduit, dans les villages-sites choisis, des diagnostics participatifs sur les conditions de production et les utilisations du sorgho (MartínezSánchez, 2003) ainsi que sur les réseaux et mécanismes d'échanges et de production de semences (Plessis, 2004). Les variétés locales collectées au cours de ces diagnostics ont été caractérisées aux plans morphologique, physiologique et agronomique. Finalement, les diagnostics et les premiers essais variétaux mis en place in situ ont conduit à la codéfinition avec les agriculteurs des objectifs d'amélioration et des critères de sélection pour quatre types de sorgho (tortillero et millón + sorghos à balais et sorghos à grain rouge). Les principaux résultats et enseignements de cette phase initiale ont été présentés dans une publication récente (Trouche et al., 2006a). La démarche et les dispositifs de mise en ouvre des activités de recherche en collaboration avec les partenaires ont été décrits et discutés dans deux autres publications (Trouche et al., 2006b ; Hocdé, 2006).

Cet article présente et analyse les résultats les plus avancés obtenus de 2002 á 2006 avec la stratégie de sélection variétale participative (participatory varietal selection, PVS) recourrant á une ample diversité de variétés et lignées sélectionnées, en majorité originaires d'Afrique de l'Ouest.

\section{Matériel et méthode}

\section{Origine et diversité du matériel végétal}

Un premier projet sur l'amélioration variétale des sorghos tortillero, conduit au Nicaragua de 1986 à 1990 par le Cirad et le ministère de l'Agriculture et de la Réforme agraire, avait abouti à la sélection de la variété IRAT 204 pour les zones sèches de basse altitude. Cette variété, développée au Sénégal, a apporté un gain important en précocité (première variété de 90 jours) et en qualité de grain. Pour ces raisons, elle a été rapidement vulgarisée au début des années 1990 sous le nom de Tortillero Precoz, et reste aujourd'hui encore la variété commerciale la plus demandée. Cette expérience positive de l'adaptation des variétés africaines de cycle court aux conditions du Nicaragua et la disponibilité de nouveaux génotypes développés pour des agrosystèmes avec contraintes de sécheresse et/ou de fertilité des sols au Mali et Burkina Faso, ont incité l'équipe de recherche à recourir largement à du germoplasme ouest-africain pour démarrer ce nouveau projet. D'autres sources de germoplasme ont été également exploitées : des cultivars locaux collectés durant le diagnostic, des lignées introduites et/ou développées par l'INTA, des lignées photopériodiques de taille courte provenant du programme INTSORMIL en Amérique centrale et des écotypes photopériodiques africains de la core collection sorgho Cirad.

\section{Méthode d'évaluation et sélection participative}

Cette stratégie de sélection, qui cherche à tirer directement profit du matériel végétal avancé existant (variétés et lignées fixées), a été mise en œuvre dès la première année du projet.

Les essais variétaux présentant aux agriculteurs, dans leur première étape, une gamme diversifiée de génotypes, sont mis en place in situ en champs paysans et gérés par des agriculteurs-expérimentateurs (AE) volontaires. Les chercheurs y mesurent les variables agronomiques et les rendements grain et paille; les producteurs de la communauté évaluent les génotypes d'abord au champ puis en postrécolte selon leurs propres critères et indiquent leurs préférences. L'analyse de ces deux types de résultats permet aux chercheurs de : i) préciser les idéotypes des agriculteurs ; ii) déterminer les génotypes qui sont á la fois performants dans les systèmes de culture cibles et qui s'approchent le plus des idéotypes définis.

Le processus formel d'évaluation et sélection participative se déroule en quatre étapes : essais préliminaires, de confirmation, prévalidation et validation.

Les évaluations participatives au champ sont réalisées au stade de maturité, généralement par de petits groupes de deux à cinq agriculteurs évaluateurs; chaque groupe évalue l'ensemble des génotypes de l'essai sur deux à trois répétitions pour 
les quatre critères de sélection de plus grande importance, préalablement identifiés par les agriculteurs du village, et pour la valeur agronomique générale, selon une échelle de valeur (score). Seuls les meilleurs génotypes au champ sont ensuite évalués par un panel de producteurs et consommateurs (hommes et femmes) pour les aspects de qualité de grain, incluant l'aptitude au battage et à l'élaboration de tortillas (suivant un protocole détaillé s'appuyant sur les méthodes locales de préparation) et l'apparence et la saveur des tortillas (au moyen de tests de dégustation).

À la fin de chaque cycle agricole, le choix des génotypes avançant á l'étape suivante est effectué de manière consensuelle entre les chercheurs et les $\mathrm{AE}$ au cours d'ateliers de discussion des résultats. Ces décisions s'appuient à la fois sur les résultats agronomiques et les appréciations des agriculteurs au champ et dans les tests de dégustation. Les génotypes ainsi retenus sont multipliés en saison sèche afin de disposer des semences nécessaires pour les essais du cycle suivant.

\section{Résultats}

\section{Dynamique du projet entre 2002 et 2006}

La dynamique des activités de sélection variétale participative entre 2002 et 2006 est résumée dans le tableau 1. L'augmentation rapide du nombre d'AE entre 2003 et 2004 traduit une implication plus forte des ONG et organisations paysannes partenaires au premier semestre 2004, surtout dans la zone Nord, qui a permis d'étendre le travail sur de nouveaux villages-sites et de mobiliser de nouveaux groupes d'agriculteurs sur les sites initiaux. Les ateliers annuels de présentation et discussion des résultats d'essais avec les AE dans chaque site ont également joué un rôle majeur dans l'engagement de nouveaux agriculteurs dans le projet.

\section{Résultats des travaux de sélection variétale participative}

Après quatre campagnes agricoles, la stratégie PVS appliquée a permis d'identifier plusieurs variétés de qualité, capables d'associer adaptation aux systèmes de culture locaux, bonnes performances

\section{Tableau 1. Évolution des activités de sélection variétale participative du sorgho conduit sur la période 2002-2006 dans deux régions du Nicaragua.}

Table 1. Development of the activities on participatory varietal selection of sorghum implemented during the 2002-2006 period in two regions of Nicaragua.

\begin{tabular}{|c|c|c|c|c|c|}
\hline Année & Type & $\begin{array}{c}\text { Étape } \\
\text { d'évaluation }\end{array}$ & $\begin{array}{c}\text { Nombre } \\
\text { de villages }\end{array}$ & $\begin{array}{c}\text { Nombre } \\
\text { d'AE }^{\prime}{ }^{1}\end{array}$ & $\begin{array}{c}\text { Nombre } \\
\text { de génotypes } \\
\text { exotiques testés }\end{array}$ \\
\hline \multirow[t]{2}{*}{2002} & Tortillero & Préliminaire & 4 & 6 & 42 \\
\hline & Millón & Préliminaire & 2 & 2 & 21 \\
\hline \multirow[t]{2}{*}{2003} & Tortillero & $\begin{array}{l}\text { Préliminaire } \\
\text { Confirmation }\end{array}$ & 6 & 13 & $\begin{array}{c}111^{*} \\
18\end{array}$ \\
\hline & Millón & Préliminaire & 4 & 5 & 36 \\
\hline \multirow[t]{2}{*}{2004} & Tortillero & $\begin{array}{l}\text { Confirmation } \\
\text { et prévalidation }\end{array}$ & 12 & 48 & 18 \\
\hline & Millón & Confirmation & 6 & 17 & 16 \\
\hline \multirow{2}{*}{$\begin{array}{l}2005- \\
2006\end{array}$} & Tortillero & Validation $^{2}$ & 12 & 32 & 4 \\
\hline & Millón & $\begin{array}{l}\text { Confirmation } \\
\text { Validation }\end{array}$ & $\begin{array}{c}9 \\
10\end{array}$ & $\begin{array}{l}20 \\
20\end{array}$ & 10 \\
\hline
\end{tabular}

${ }^{1}$ agriculteurs-expérimentateurs (AE) ayant conduit un essai complet ou une répétition d'un essai jusqu'à son terme.

2 Évaluations en parcelles de production de $500 \mathrm{~m}^{2}$ ou plus.

*Évaluation conduite en station.

agronomiques et qualité de grain et/ou paille recherchée par les producteurs.

Pour le type tortillero, la variété Cirad 492 (BF 89-12/1-1-1) cosélectionnée avec les groupes d'agriculteurs de la zone Nord, présente d'excellents résultats dans les systèmes de culture à faibles intrants non mécanisés des zones les plus sèches (<400 mm durant le cycle de culture). Dans les essais de validation en grandes parcelles et sous gestion paysanne (sans ou très faible fertilisation), elle surpasse de 43 et $36 \%$ en rendement la variété témoin des producteurs, respectivement pour 16 localités de la région Nord et 21 localités des zones Nord, Centre et Pacifique sud (figure 1). Elle est également très appréciée par les producteurs pour sa grande précocité et son excellente qualité de grain pour l'élaboration de tortillas et la vente (tableau 2).

Pour le type millón, les variétés photopériodiques PI 569438 (Soudan), 99 PREEIME 119 et EIME 119 (El Salvador), ont démontré une bonne adaptation aux conditions édapho-climatiques et aux systèmes de culture associée millón-maïs des zones de collines et apportent des progrès intéressants par rapport aux cultivars locaux pour le rendement et la qualité de grain (tableau 3). Il en est de même pour deux autres variétés d'introduction plus récente, 1583 (Cameroun, à grain orangé) et 1577 (Tchad).

Deux variétés de sorgho à balais (origines Hongrie et États-Unis), présentant une meilleure production de fibres longues et plus résistantes aux maladies foliaires que les cultivars locaux et trois lignées á grain rouge (origine Burkina), précoces et d'excellente productivité en grain et fourrage, ont été également cosélectionnées avec les agriculteurs.

\section{Discussion}

Depuis le milieu des années 1990, de nombreux travaux de recherche ont démontré l'efficacité de la sélection variétale participative décentralisée pour identifier des variétés plus performantes et mieux acceptées par les producteurs pour des environnements à fortes contraintes, chez le sorgho (Mulatu et Belete, 2001), le riz (Joshi et Witcombe, 1996; Trouche et al., 2006c), l'orge (Ceccarelli et al., 2000). Ce projet sorgho conduit au Nicaragua aboutit à des résultats similaires. En s'intégrant dans les systèmes de culture existants, en apportant des gains de productivité significatifs ainsi que d'autres caractéristiques importantes, les variétés Cirad 492, 99 PREEIME 119 et EIME 119 sont aptes à être inscrites au catalogue national (Cirad 492 a été inscrite fin 2007 sous le nom de Blanco Tortillero) et à être rapidement diffusées. L'inscription d'une nouvelle variété étant un processus relati- 


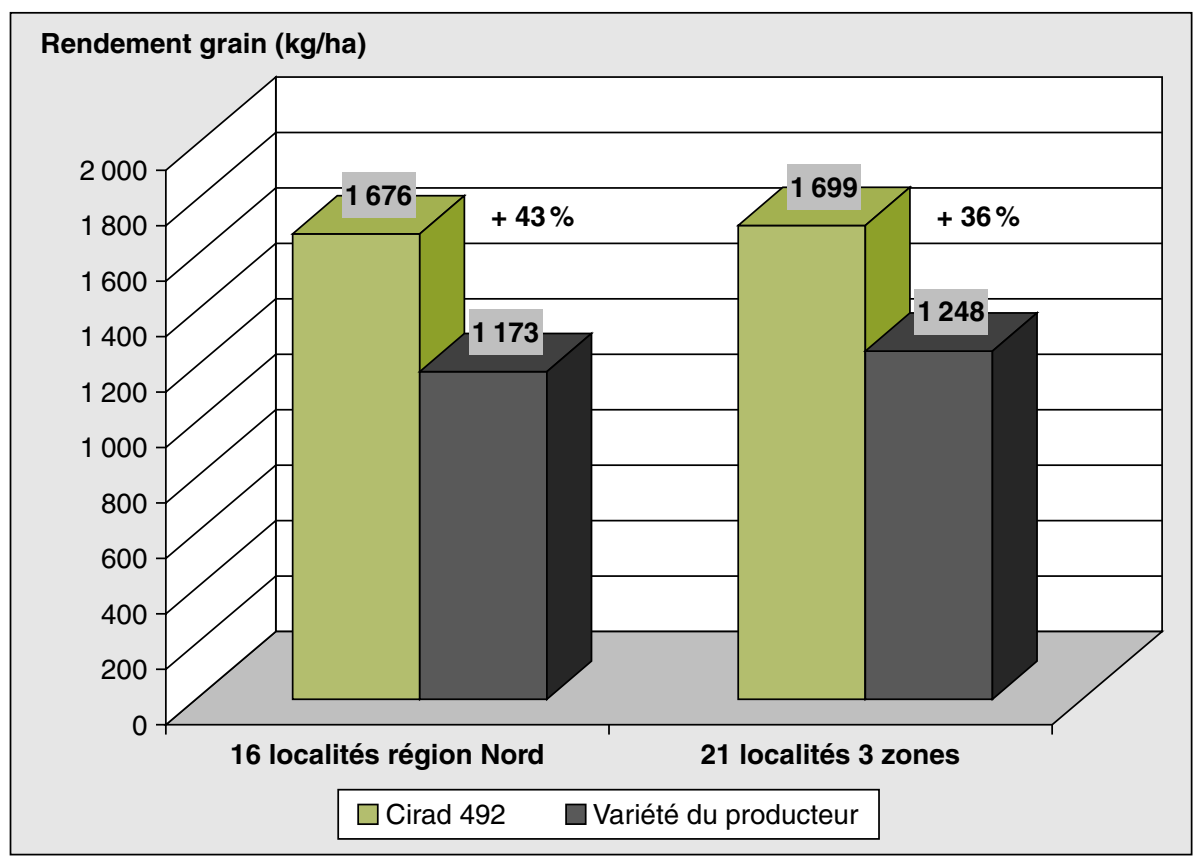

Figure 1. Gains de rendement grain obtenus par la variété Cirad 492 en comparaison aux variétés des producteurs dans les essais de validation.

Figure 1. Grain yield gains obtained by the variety Cirad 492 compared with the farmers' varieties in the pre-launching trials.

vement long et onéreux, il ne concernera, dans l'immédiat, que les génotypes à plus large adaptation. Dans le cas de la variété PI 569438, très appréciée par les producteurs de la région Nord pour son adaptation aux sols pauvres, tolérance à la sécheresse et qualité de grain (mais non acceptée dans la région Centre à cause de sa haute taille), les producteurs n'ont pas souhaité attendre une éventuelle inscription pour l'utiliser. Dès 2005, une diffu- velle variété ${ }^{1}$. Avec la démarche de sélection variétale participative décentralisée, la diffusion des nouvelles variétés a débuté quatre ans après les premiers essais in situ, d'où un gain de temps de trois ans par rapport au schéma conventionnel.

\section{Conclusion}

De l'expérience acquise avec ce projet, on peut tirer les enseignements suivants pour l'amélioration variétale des sorghos destinés aux petits producteurs et agrosystèmes à faibles intrants des zones sèches d'Amérique centrale:

- les sorghos africains introduits permettent d'élargir la diversité génétique de travail et l'offre variétale disponible dans la région centro-américaine ;

- l'introduction de lignées améliorées d'origine ouest-africaine a permis des avancées significatives pour les sorghos de type tortillero (cas d'IRAT 204 dans les années 1990 et Cirad 492 actuellement).

Certaines lignées sélectionnées dans les conditions de culture sahéliennes sont donc performantes et appréciées au Nicaragua, grâce à des caractéristiques bien identifiées comme la rusticité, le rendement, un cycle plus court, le type de grain. De surcroit, elles ont toutes les chances d'être facilement adoptées, car au-delà de leurs performances agronomiques, elles s'inscrivent bien dans les systèmes de culture locaux ;

Avec le schéma conventionnel d'évaluation variétale appliqué par le programme sorgho de l'INTA, il faut compter au minimum sept ans pour vulgariser une nou-

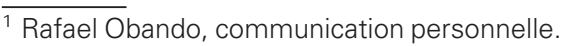

\section{Tableau 2. Performances agronomiques et appréciations par les agriculteurs de la variété de sorgho tortillero Cirad 492 cosélectionnée avec les agriculteurs dans la région Nord.}

Table 2. Agronomic performance and farmer appreciation of the tortillero sorghum variety Cirad 492 co-selected with the farmers in the Northern region.

\begin{tabular}{|c|c|c|c|c|c|c|c|c|}
\hline \multirow[t]{2}{*}{ Variété } & \multirow{2}{*}{$\begin{array}{c}\text { Rendement } \\
\text { grain (kg/ha) } \\
\text { en essais de } \\
\text { validation ( } 21 \\
\text { localités } \\
\text { en } 2005 \text { et } 2006 \text { ) }\end{array}$} & \multirow{2}{*}{$\begin{array}{c}\text { Rendement } \\
\text { grain (kg/ha) } \\
\text { en essais } \\
\text { de confirmation } \\
\text { (10 essais } \\
\text { de } 2002 \text { à 2004) }\end{array}$} & \multirow{2}{*}{$\begin{array}{l}\text { Duré } \\
\text { du cycle } \\
\text { semis- } \\
\text { floraison } \\
\text { (jours) }\end{array}$} & \multirow{2}{*}{$\begin{array}{l}\text { Poids } \\
\text { de } 1000 \\
\text { grains (g) }\end{array}$} & \multicolumn{4}{|c|}{ Appréciation des agriculteurs } \\
\hline & & & & & $\begin{array}{c}\text { Type } \\
\text { de grain }{ }^{1}\end{array}$ & $\begin{array}{c}\text { Valeur } \\
\text { générale }\end{array}$ & FSP $(\%)^{2}$ & $\begin{array}{c}\text { Rendement } \\
\text { et qualité } \\
\text { des tortillas }\end{array}$ \\
\hline Cirad 492 & $1699 a^{4}$ & $2380 a^{4}$ & $60 \pm 2,5$ & $30 \pm 2,7$ & 3,2 & 3,3 & 94 & 2,9 \\
\hline $\begin{array}{l}\text { Tortillero } \\
\text { Precoz }\end{array}$ & - & $1790 \mathrm{~b}$ & $59 \pm 2,1$ & $27 \pm 4,1$ & 2,8 & 2,9 & 42 & n.e. \\
\hline Tortillero local & $1248 b$ & - & $63 \pm 2,7$ & $30 \pm 3,9$ & 3,2 & 3 & 64 & 2,8 \\
\hline
\end{tabular}

${ }^{1}$ Note moyenne d'appréciation de la variété par les agriculteurs évaluateurs pour un critère donné au cours des évaluations participatives au champ, selon l'échelle de notation suivante 1 = mauvais ; 2 = moyen ; 3 = bon ; 4 = excellent

${ }^{2} \mathrm{FSP}=$ fréquence de sélection de la variété par les agriculteurs au cours des évaluations participatives au champ.

${ }^{3}$ Note moyenne d'aptitude à la préparation et de qualité des tortillas selon 7 critères dans les tests de dégustation in situ; même échelle de notation que pour l'évaluation au champ.

${ }^{4}$ Test de comparaison des moyennes de Newman-Keuls : les moyennes ayant la même lettre ne sont pas significativement différentes au seuil a $=5 \%$. 
Tableau 3. Performances agronomiques et appréciation par les agriculteurs de trois lignées de sorgho millón cosélectionnées avec les agriculteurs dans les régions Nord et Centre.

Table 3. Agronomic performance and farmer appreciation of three millon sorghum lines co-selected with the farmers in the Northern and Central regions.

\begin{tabular}{|c|c|c|c|c|c|c|c|c|}
\hline \multirow[t]{2}{*}{ Lignée } & \multicolumn{2}{|c|}{$\begin{array}{c}\text { Rendement grain ( } \mathrm{kg} / \mathrm{ha}) \\
\text { dans essais de validation } \\
(12 \text { localités } \\
\text { en } 2005 \text { et } 2006)\end{array}$} & \multirow[t]{2}{*}{$\begin{array}{l}\text { Durée du cycle } \\
\text { semis-floraison } \\
\text { (jours) }\end{array}$} & \multirow[t]{2}{*}{$\begin{array}{l}\text { Hauteur } \\
\text { de plante } \\
\text { (cm) }\end{array}$} & \multirow[t]{2}{*}{$\begin{array}{l}\text { Poids } \\
\text { de } 1000 \\
\text { grains (g) }\end{array}$} & \multicolumn{3}{|c|}{$\begin{array}{l}\text { Appréciation } \\
\text { des agriculteurs }\end{array}$} \\
\hline & Moyenne & $\Delta \mathbf{T}^{1}$ & & & & $\begin{array}{c}\text { Type } \\
\text { de grain }^{3}\end{array}$ & $\begin{array}{c}\text { Valeur } \\
\text { générale }\end{array}$ & FSP $(\%)^{4}$ \\
\hline 99 PREEIME 119 & 1767 & $1,21 \mathrm{~ns}$ & 164 & $208 \pm 30,0$ & $26 \pm 3,2$ & 3 & 3,4 & 85 \\
\hline PI 569438 & 1657 & $0,95 \mathrm{~ns}$ & 162 & $344 \pm 42,7$ & $37 \pm 3,0$ & 3,5 & 2,9 & 67 \\
\hline EIME 119 & 1672 & $0,91 \mathrm{~ns}$ & 161 & $202 \pm 21,0$ & $26 \pm 3,3$ & 3,2 & 3,5 & 89 \\
\hline $\begin{array}{l}\text { MILLON LOCAL } \\
\text { (variété témoin) }\end{array}$ & 1447 & & 165 & $286 \pm 10,3$ & $27 \pm 1,9$ & 3 & 2,7 & 7 \\
\hline
\end{tabular}

${ }^{1}$ Valeur de t pour la différence de rendement avec la variété locale du producteur ; ns = non significatif.

2 Pour une date de semis au 5 juin.

${ }^{3}$ Note moyenne d'appréciation de la variété par les agriculteurs évaluateurs pour un critère donné au cours des évaluations participatives au champ (étape essais de confirmation), selon l'échelle de notation suivante : $1=$ mauvais $; 2=$ moyen $; 3=$ bon ; 4 = excellent.

${ }^{4} \mathrm{FSP}=$ fréquence de sélection de la variété par les agriculteurs au cours des évaluations participatives au champ.

\section{Tableau 4. Principales étapes des progrès réalisés dans l'amélioration variétale des sorghos au Nicaragua.}

Table 4. Main stages of the progress achieved for sorghum improvement in Nicaragua.

\begin{tabular}{|c|c|c|c|}
\hline Périodes & Variétés & $\begin{array}{c}\text { Origine } \\
\text { du matériel génétique }\end{array}$ & $\begin{array}{l}\text { Modes d'obtention } \\
\text { et de sélection }\end{array}$ \\
\hline Avant années 1950 & $\begin{array}{l}\text { Uniquement variétés criollas de type } \\
\text { millón } \\
\text { Rendement moyen }{ }^{1}: 0,7-1,2 \mathrm{t} / \mathrm{ha}\end{array}$ & Afrique australe? & Sélection paysanne \\
\hline $\begin{array}{l}\text { 1970-1980: } \\
\text { premières } \\
\text { sécheresses }\end{array}$ & $\begin{array}{l}\text { Premières variétés améliorées de cycle } \\
\text { court, type tortillero: Guatecao, Shallu... } \\
\text { Rendement moyen }{ }^{1}: 1-1,5 \text { t/ha }\end{array}$ & $\begin{array}{l}\text { Amérique centrale } \\
\text { et États-Unis }\end{array}$ & $\begin{array}{l}\text { Introduction et diffusion } \\
\text { sans évaluation formelle }\end{array}$ \\
\hline 1982-1985 & $\begin{array}{l}\text { Variétés améliorées " salvadoriennes ": } \\
\text { Tortillero, Ligero, Blanco, Estopa Negra } \\
\text { (100-110 jours) } \\
\text { Rendement moyen" : 1,2-2 t/ha }\end{array}$ & Salvador & $\begin{array}{l}\text { Introduction par ONG } \\
\text { et commerçants et diffusion } \\
\text { par réseaux informels }\end{array}$ \\
\hline $1987-1990$ & $\begin{array}{l}\text { IRAT } 204 \text { (Tortillero Precoz, } 90 \text { jours) et } \\
\text { SPV } 475 \text { (Pinolero 1, } 110 \text { jours) } \\
\text { Rendements moyens }{ }^{1}: 1,5-2,5 \text { t/ha } \\
\text { Rendement potentiel }{ }^{2} \text { (TP) : } 5 \text { t/ha }\end{array}$ & Afrique de l'Ouest et Inde & $\begin{array}{l}\text { Introduction et processus } \\
\text { d'évaluation et sélection } \\
\text { conventionnels }\end{array}$ \\
\hline 2002-2007 & $\begin{array}{l}\text { Cirad } 492 \text { (Blanco Tortillero, } 90 \text { jours) } \\
\text { Rendements moyens } 1: 1,7-3 \text { t/ha } \\
\text { Rendement potentiel }\left.\right|^{2}: 6 \text { t/ha }\end{array}$ & $\begin{array}{l}\text { Afrique de l'Ouest } \\
+ \text { autres régions }\end{array}$ & $\begin{array}{l}\text { Introduction et processus } \\
\text { d'évaluation et sélection } \\
\text { participatifs décentralisés }\end{array}$ \\
\hline 2006-2010? & $\begin{array}{l}\text { Lignées dérivées des croisements CIR-1, } \\
\text { CIR-2 et CIR-6 } \\
\text { Rendements potentiels }{ }^{2}: 6-8 \text { t/ha }\end{array}$ & $\begin{array}{l}\text { Croisements variétés locales } \\
\text { x lignées améliorées ouest- } \\
\text { africaines }\end{array}$ & $\begin{array}{l}\text { Création variétale } \\
\text { participative décentralisée }\end{array}$ \\
\hline
\end{tabular}

${ }^{1}$ Rendements moyens en milieu réel sous pratiques paysannes dans les zones sèches du Nord et Centre.

${ }^{2}$ Rendements maxima obtenus en essais sous gestion chercheur sur la station de recherche du Centro Nacional de Investigación Agropecuaria (CNIA) près de Managua. 
- existe-t-il encore une marge de progrès génétique importante pour ces conditions de culture à faibles intrants du Nicaragua? Les premiers résultats d'évaluation de 120 nouvelles lignées de type tortillero issues de schémas de création variétale participatifs in situ, conduits depuis 2003 à partir de croisements entre des cultivars locaux bien appréciés et des lignées sélectionnées ouest-africaines, semblent apporter une réponse positive;

- si l'on porte un regard sur les progrès réalisés en matière de variétés de sorgho au cours des 50 dernières années (tableau 4), on observe qu'ils sont le résultat d'un ensemble de facteurs combinant l'origine du germoplasme et la stratégie de sélection, dont la dernière étape est la création variétale in situ à partir de croisements raisonnés entre agriculteurs et chercheurs ;

- cela étant, autant sélectionner parmi des variétés ou lignées fixées n'est pas trop difficile ni contraignant pour les agriculteurs, autant travailler à la sélection de plantes dans des populations en ségrégation durant plusieurs cycles requiert de nouvelles compétences et une vision à plus long terme. Cela demande également de nouveaux modes d'interactions entre chercheurs et agriculteurs pour inscrire ce type de recherche dans la durée.

\section{Références}

Ceccarelli S, Grando S, Tutwiler R, et al. () A methodological study on participatory barley breeding. I. Selection phase. Euphytica 2000(111) : 91-104.

Hocdé $\mathrm{H}$. Fitomejoramiento participativo de cultivos alimenticios en Centro América panorama, resultados y retos. Un punto de vista externo. Agronomía Mesoamericana 2006 ; $17: 291-308$.

Instituto nacional de estadisticas y censos de Nicaragua (Inec). Resultados del Tercer Censo Nacional Agropecuario (III CENAGRO). Managua : Inec, 2002. Consultable en ligne : www.i nec.gob.ni.

Joshi A, Witcombe JR. Farmer participatory crop improvement. II. Participatory varietal selection, a case study in India. Exp Agric $1996 ; 32: 461-77$.

Martínez Sánchez F. Análisis de los sistemas de cultivo a base de sorgo para la construcción de un programa de mejoramiento genético participativo en el departamento de Madriz, Nicaragua. Mémoire de Master of Science en "Développement Agricole Tropical", Cnearc Montpellier, 2003.
Mulatu E, Belete K. Participatory varietal selection in lowland sorghum in eastern Ethio pia : impact on adoption and genetic diversity. Exp Agric $2001 ; 37$ : 211-29.

Plessis J. Vers la compréhension de la gestion locale des semences de sorgho: une aide à la diffusion des variétés générées par un programme de sélection participative. Etude réalisée dans la zone nord du Nicaragua. Mémoire d'ingénieur Istom, Cergy-Pontoise, France, 2004.

Trouche G, Hocdé H, Aguirre Acuña S, Martínez Sánchez F, Gutiérrez Palacios N. Dinámicas campesinas y fitomejoramiento participativo: el caso de los sorgos blancos (Sorghum bicolor, (L). Moench) en la region Norte de Nicaragua. Agronomía Mesoamericana (CR) 2006a ; $17:$ 407-25.

Trouche G, Hocdé H, Aguirre Acuña S. Sélection participative des sorghos au Nicaragua : approche et dispositifs. In : Lançon J, Floquet A, Weltzien E, eds. Partenaires pour construire des projets de sélection participative. Actes de l'atelier-recherche, 14-18 mars 2005. Cotonou, Bénin. Montpellier: Cirad éditions, Inrab, Coopération française, $2006 \mathrm{~b}$.

Trouche G, Narváez Rojas L, Chow Wong Z, Corrales Blandón J. Fitomejoramiento participativo del arroz de secano en Nicaragua: metodologias, resultados y lecciones aprendidas. Agronomía Mesoamericana (CR) 2006c $17: 309-25$. 